\title{
Time to Frequency Domain Analysis of Narrow Band Antennas
}

\author{
Sajjad Ur Rehman and Majeed A. S. Alkanhal \\ Department of Electrical Engineering, King Saud University, Riyadh, Saudi Arabia \\ Correspondence should be addressed to Majeed A. S. Alkanhal; majeed@ksu.edu.sa
}

Received 25 January 2017; Revised 21 March 2017; Accepted 5 April 2017; Published 30 April 2017

Academic Editor: Ikmo Park

Copyright (C) 2017 Sajjad Ur Rehman and Majeed A. S. Alkanhal. This is an open access article distributed under the Creative Commons Attribution License, which permits unrestricted use, distribution, and reproduction in any medium, provided the original work is properly cited.

\begin{abstract}
This paper presents system modeling of three different single band antennas using the singularity expansion method (SEM). To acquire the accurate physical complex poles and the corresponding residues of the narrow band antenna model, an enhanced Matrix Pencil (MP) method has been applied to the impulse response of the antennas. A delayed time parameter $T$ is introduced to enhance the performance of the MP method to extract the actual physical poles and eliminate the nonphysical poles caused by the early time response of the narrow band antennas. The poles extracted by the enhanced MP method are located precisely within the single operating band of each antenna. The one set of the developed parameters is used to accurately characterize narrow band antennas in both time and frequency domains. The far field impulse response of a fabricated single band microstrip antenna has been measured in the laboratory and then reconstructed precisely using the SEM based model with the proposed MP scheme.
\end{abstract}

\section{Introduction}

Since the introduction of the singularity expansion method (SEM) [1], it has been widely used in the analysis of transient electromagnetic scattering and radar target recognition [24]. The SEM is a physical system model that describes the transient electromagnetic phenomena in time and frequency domain. The SEM provides solution for electromagnetic interaction problems in terms of singularity poles in the complex frequency domain [5]. Recently, SEM has been applied to medical diagnosis signals to estimate the diameter of the stent during and after deployment in human artery [6]. In recent few years SEM has been applied effectively to characterize ultra-wide band (UWB) antennas in both time and frequency domains [7-9].

One of the main restrictions in SEM based models is its total dependence on the accuracy of the extracted complex poles and their corresponding residues. UWB antennas have been efficiently characterized using SEM based model in the past. The UWB antennas have rather wide operating bands, and then, all the extracted poles and their response are within the UWB. In the case of narrow band antennas, the limited extracted in-band poles are very sensitive to the out-band poles and hence, the antenna response is typically corrupted by the contribution of the out-band poles and their energies. Therefore, it is quite a challenge to develop a narrow band antenna system model based on SEM.

To extract the SEM parameters, a mathematical method is needed to extract the poles and their residues efficiently. There are various methods, like Prony's, Cauchy's, and Matrix Pencil (MP) method, utilized for poles and residues extraction. Among these, Matrix Pencil (MP) [10] method is known to be more efficient [7].

In the field of antennas and scattering, SEM has been applied positively in both time and frequency domains to model UWB antenna's effective length in order to fully describe the antenna radiation patterns, directivity, and gain [8]. The poles of the effective length were extracted using the MP method. The method established in [8] is merely accurate in modeling UWB antennas. In addition, MP has been applied successfully in [11] to extract complex natural resonance (CNR) poles of RFID tags from the encoded data on the tags.

In this paper, an enhanced Matrix Pencil (MP) scheme is presented in order to extract poles accurately for narrow band antennas. From careful analysis of the antenna far field impulse response, it is noticed that the early time response of the antenna contributes nonphysical complex poles with low 


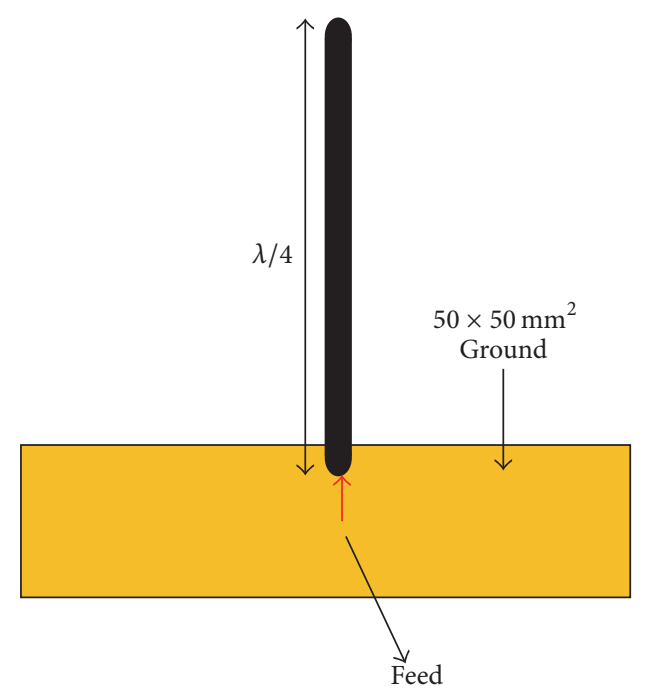

Figure 1: Geometry of the $\lambda / 4$ wire-monopole antenna.

energies. Apparently, this is a major problem in developing the SEM model for narrow band antennas. To overcome this effect, we introduced an additional delayed time parameter $T$ in the MP scheme to sift out the early time response and its low energy complex poles. In this paper, the conventional MP method is applied first to three different single band antennas (wire-monopole, microstrip, and coplanar waveguide-CPW antennas). To discriminate between physical and nonphysical poles, the energy of each extracted pole pair is deliberated. The enhanced MP method with the time parameter $T$ is then applied to all the three mentioned antennas. The results for the classical MP method and for the enhanced scheme with the delayed time parameter $T$ are compared. To validate the accuracy of the extracted poles, the original impulse response is reconstructed using SEM. Section 3 of this paper includes the description of the enhanced MP scheme. In Section 4, the classical and the enhanced MP schemes are applied to the impulse responses of the three different single band antennas and the results are compared. Section 5 discusses measured results.

\section{The Singularity Expansion Method (SEM)}

The SEM permits modeling of the late time domain response of an antenna as an exponential sum with a set of parameters as [5]

$$
x(t)=\sum_{i=1}^{M} R_{i} e^{s_{i} t},
$$

where $x(t)$ is the antenna response, $s_{i}$ is the $i$ th pole, $R_{i}$ is the corresponding residue of the $i$ th pole, and $M$ is the number of poles. Every pole is defined as $s_{i}=\alpha_{i}+j \omega_{i}$, where $\alpha_{i}$ is the damping coefficient of the $i$ th pole and $\omega_{i}$ is the complex resonant frequency of the $i$ th pole. In frequency domain, SEM allows modeling the transfer function $X(S)$ of the antenna as [5]

$$
X(S)=\sum_{i=1}^{M} \frac{R_{i}}{s-s_{i}},
$$

where $S$ is the Laplace complex variable. There are different methods to extract the complex poles and their corresponding residues from antenna impulse response. Here in this paper, we focus on the MP method.

\section{Enhancement of the MP Method}

In time domain, the transient response can be divided into early and late time. In general, resonant modes will start to establish and stabilize in later time $[12,13]$. In this work, the MP method is implemented in this paper with a delayed time parameter $T$ to enhance the performance of extraction of the physical poles of narrow band antennas. The delayed time is processed in order to avoid the nonphysical poles caused by the early time response of the antenna. The delayed time parameter $T$ can be approximated as

$$
T \approx \frac{L}{c}+T_{P}
$$

where $L$ is the largest dimension of the antenna, $c$ is the speed of light in free space, and $T_{P}$ is the pulse duration.

The far field impulse response of the monopole antenna (shown in Figure 1) with the appropriate $T$ parameter is shown in Figure 2. In the time axis of the impulse response, the period before the time marker $T$ is the early time response with several low energy nonphysical poles.

The low energy poles are placed outside the resonance of the antenna and can be eliminated as they are not physical poles of the antenna. The poles with low energies are mainly because of smaller singular values that might be generated 


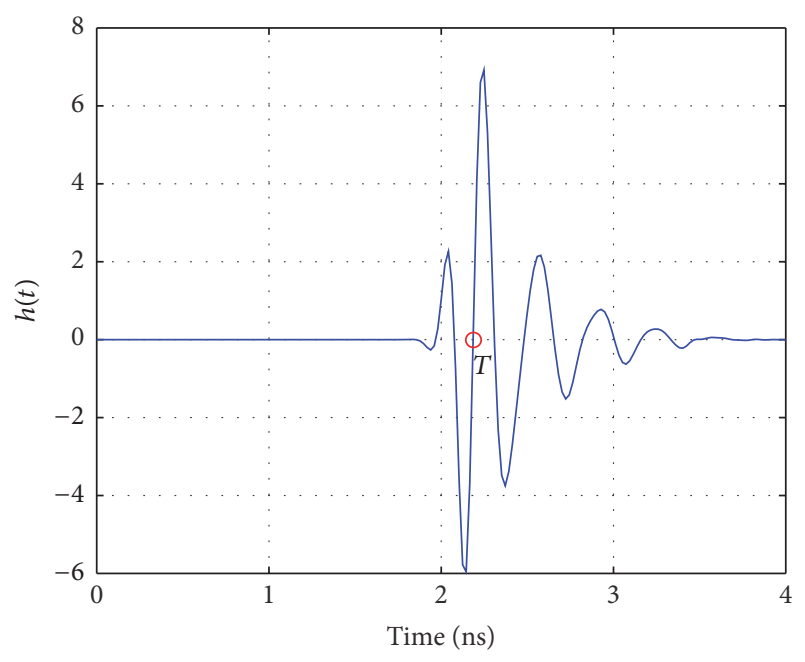

FIGURE 2: Impulse response of monopole antenna with the delayed time parameter $T$ indicated.

due to some numerical or measurement noise. The energy of pole pair $(E)$ is proportional to the square of the magnitude of the pole and inversely proportional to the damping factor [11]:

$$
E=\frac{R^{2}}{\text { Damping Factor }}
$$

Typically, the low energy poles have lower residues and higher damping factors.

\section{System Models of Narrow Band Antennas}

The classical MP and the enhanced MP schemes are applied to the impulse responses of three single band antennas. A wiremonopole, a microstrip, and a coplanar waveguide (CPW) antennas are analyzed in this section. All the antennas are simulated using the CST Microwave Studio [14]. The time domain solver of the CST Microwave Studio is used to obtain the simulated far field impulse responses of all antennas. The results of both MP schemes are compared and the original impulse responses of all antennas are reconstructed at the end to validate the accuracy of the extracted poles.

4.1. SEM Model for the Wire-Monopole. Figure 1 depicts the geometry of a quarter-wave-length $(\lambda / 4)$ wire-monopole antenna. The quarter-wave-length wire-monopole antenna is designed to operate at $3.2 \mathrm{GHz}$ with radius of $0.1 \mathrm{~mm}$. $S_{11}$ of the designed antenna is shown in Figure 3 while the far field impulse response of the antenna at bore sight is shown in Figure 2. The impulse response of the monopole antenna is directly applied to the classical MP method. The pole-residue relationship and the frequency as a function of the damping factor are plotted in Figures 4(a) and 4(b), respectively.

It can be seen from Figure 4 that there are several pairs of poles located at different frequencies with a single pole located at zero. To discriminate between physical and nonphysical poles, $E$ levels of all extracted poles are assessed.



FIGURE 3: $S_{11}$ of the quarter-wave-length wire-monopole antenna.



(a)

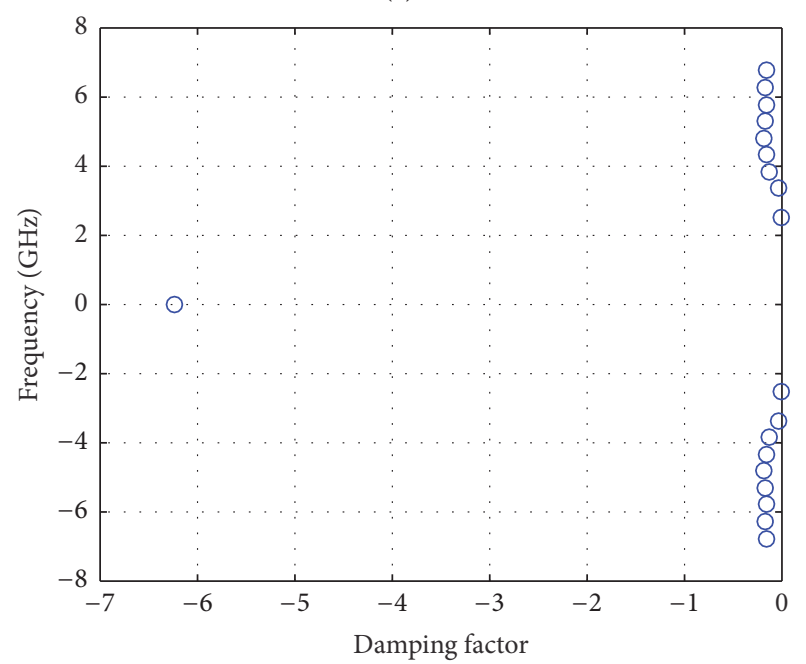

(b)

FIGURE 4: Using classical MP method: (a) pole-residue relationship and (b) frequency versus damping factor of the monopole antenna.

The normalized values of $E$ are plotted with frequency in Figure 5. 


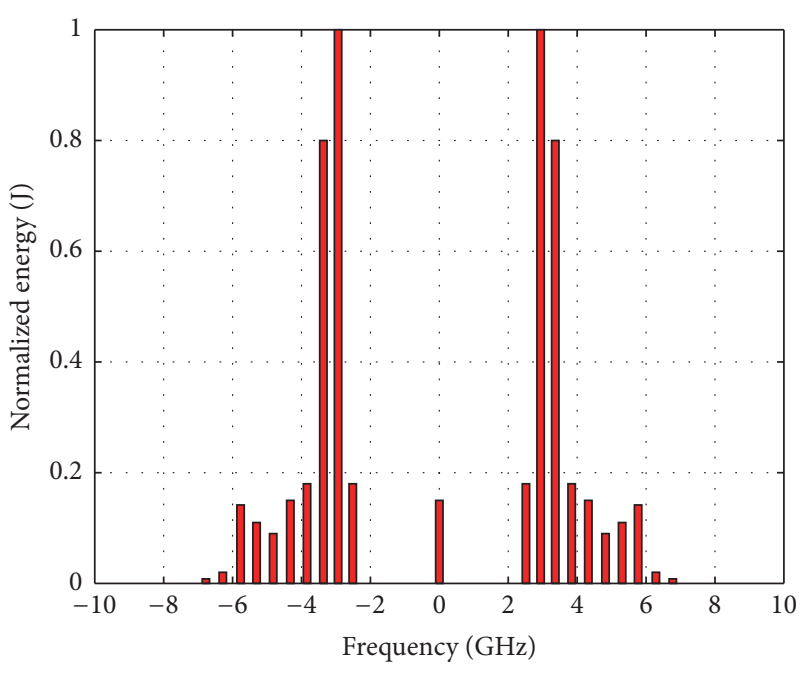

FIgURE 5: Poles normalized $E$ levels with frequency.

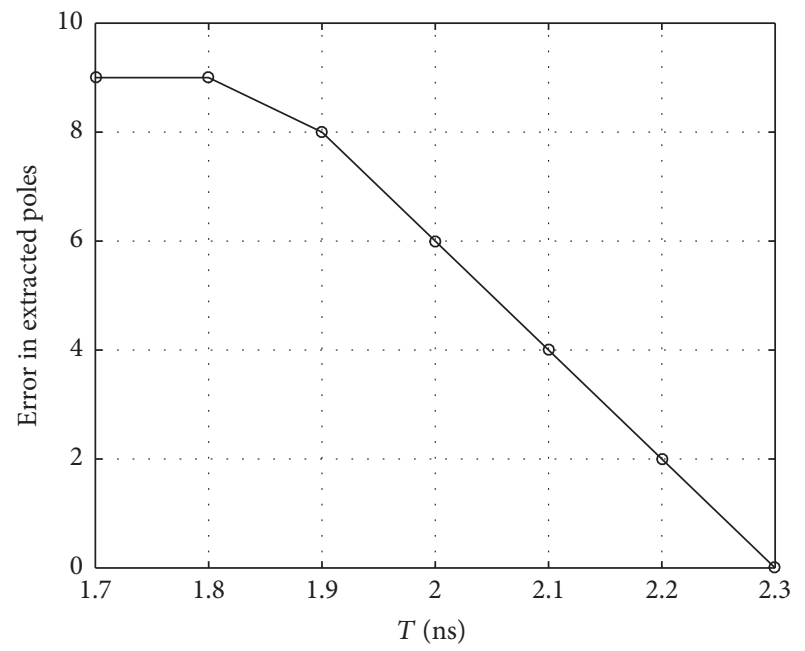

Figure 6: Error in the extracted poles as a function of the delayed time parameter $T$.

The poles located from about $2.5 \mathrm{GHz}$ up to $7 \mathrm{GHz}$ have different $E$ levels as shown in Figure 5. It can be observed from Figure 5 that there are two pairs of physical poles located at the antenna resonant frequency $(3.2 \mathrm{GHz})$ with high energy as compared to the other eight pairs of poles.

Once the number of physical poles is known then the appropriate $T$ can be estimated recursively from the MP algorithm with the aid of (3) in order to, precisely, extract physical poles of the antenna. Figure 6 illustrates the error in the extracted poles with the parameter $T$ swept across the time axis.

The impulse response shown in Figure 1 with $T=2.3 \mathrm{~ns}$ is directly applied to the code developed to implement the proposed enhanced MP scheme. The pole-residue relationship with frequency as a function of the damping factor is plotted in Figures 7(a) and 7(b), respectively. The results in Figure 7 show that the zero frequency pole and all of the low $E$ nonphysical poles in the antenna response are eliminated. There are two pairs of poles located at about



(a)

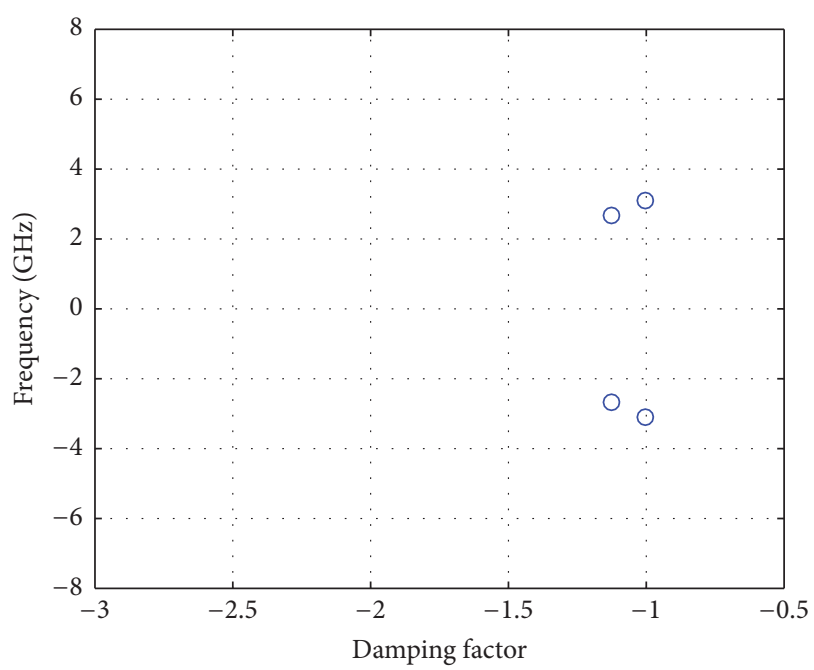

(b)

FIGURE 7: Using the proposed enhanced MP method: (a) poleresidue relationship and (b) frequency versus damping factor.

TABle 1: Parameters of the CPW antenna.

\begin{tabular}{lccc}
\hline$r(\mathrm{~mm})$ & $L_{f}(\mathrm{~mm})$ & Substrate height $(\mathrm{mm})$ & $W_{s}(\mathrm{~mm})$ \\
\hline 16 & 38 & 1.54 & 0.375 \\
\hline
\end{tabular}

3.05 GHz and 3.2 GHz. The extracted poles are well within the resonance band of the narrow band antenna. To validate the two extracted poles, the impulse response of the antenna is reconstructed using (1). Comparison of the original impulse response and the reconstructed response is shown in Figure 8 where the reconstructed response overlaps the original impulse response.

4.2. SEM Model for the CPW Antenna. A single band CPW antenna is designed on a dielectric substrate with dielectric constant of 4.3 to operate at $3 \mathrm{GHz}$. The parameters of the antenna are listed in Table 1, whereas the geometry of the antenna is shown in Figure 9. The antenna is fed through a $50 \Omega \mathrm{CPW}$ line. The simulated $S_{11}$ and the far field impulse 


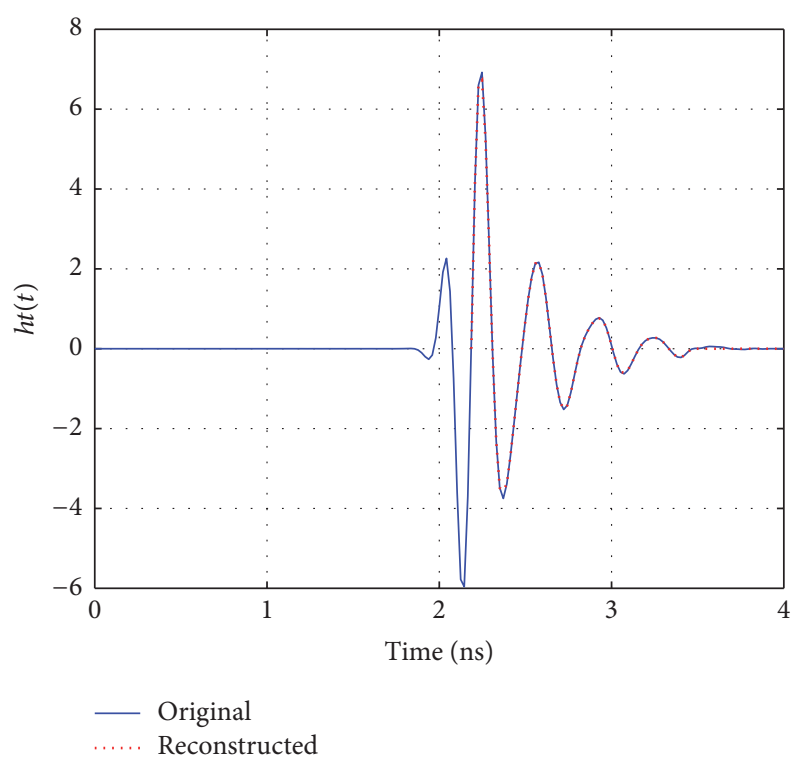

Figure 8: Comparison of the original impulse response and the reconstructed response of the wire-monopole antenna.

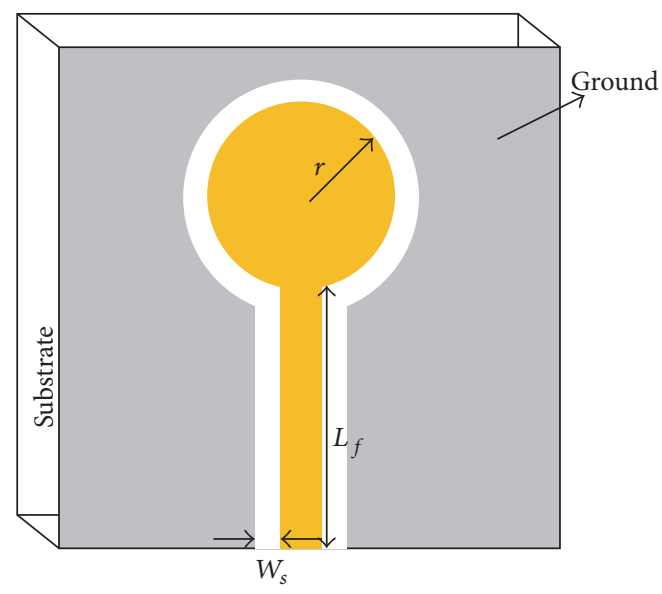

Figure 9: Geometry of the CPW antenna.

response of the antenna are shown in Figures 10 and 11, respectively.

The impulse response of the CPW antenna shown in Figure 11 is applied to the classical MP method. The poleresidue relationship and the frequency as a function of the damping factor are plotted in Figures 12 (a) and 12(b), respectively. It can be seen from Figure 12 that there are three pairs of poles located at different frequencies. $E$ of all the extracted poles are calculated and shown in Figure 13.

The pairs of poles located at the antenna resonant frequency $(3 \mathrm{GHz})$ have high energy as compared to the other two pairs of poles. Subsequently, the impulse response shown in Figure 11 is applied to the code developed to implement the proposed enhanced MP scheme. The delayed time parameter $T$ is set to $2.7 \mathrm{~ns}$. The pole-residue relationship and frequency as a function of the damping factor are plotted in Figures 14(a) and $14(\mathrm{~b})$, respectively.
TABLE 2: Parameters of the microstrip antenna.

\begin{tabular}{lcccc}
\hline$r(\mathrm{~mm})$ & $L_{f}(\mathrm{~mm})$ & Substrate height $(\mathrm{mm})$ & $S_{1}(\mathrm{~mm})$ & $S_{2}(\mathrm{~mm})$ \\
\hline 18 & 30 & 0.78 & 0.5 & 10 \\
\hline
\end{tabular}

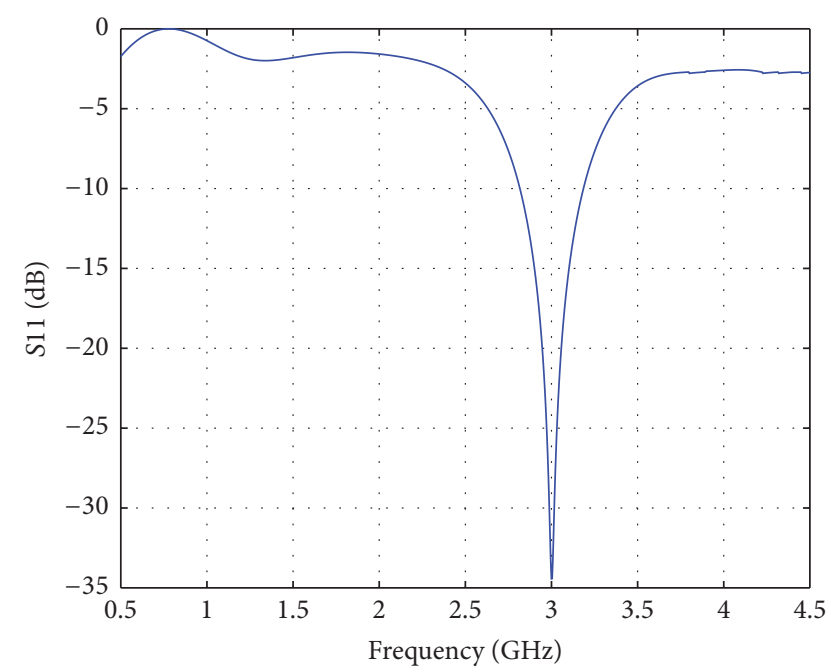

Figure 10: $S_{11}$ of the single band CPW antenna.

The results in Figure 14 show that all of the low $E$ nonphysical poles in the antenna response are eliminated. There are two pairs of poles located at about $3.0 \mathrm{GHz}$. The extracted poles are well within the resonance band of the narrow band antenna. To validate the extracted poles, the impulse response of the antenna is reconstructed using SEM (1). Comparison of the original impulse response and the reconstructed response is shown in Figure 15 where the reconstructed response overlaps the original impulse response.

4.3. SEM Model for the Microstrip Antenna. A single band microstrip circular patch antenna is designed on a dielectric substrate with dielectric constant of 4.3 to operate at $2.3 \mathrm{GHz}$. The parameters of the antenna are listed in Table 2, whereas the geometry of the antenna is shown in Figure 16. The antenna is fed through a $50 \Omega$ microstrip line. The simulated $S_{11}$ and impulse response of the antenna are shown in Figures 17 and 18, respectively.

Using the classical MP method, the pole-residue relationship and the frequency as a function of the damping factor are plotted in Figures 19(a) and 19(b), respectively. In Figure 19, there are four pairs of poles located at different frequencies. The $E$ levels of the extracted poles with frequency are shown in Figure 20.

The four pairs of poles located from about $2 \mathrm{GHz}$ up to 2.7 GHz have different $E$ levels as shown in Figure 20. The pair of poles located at the antenna resonant frequency $(2.3 \mathrm{GHz})$ has high energy as compared to the other three pairs of poles.

To get the accurate pairs of poles for this narrow band antenna and eliminate the early time nonphysical poles, the impulse response shown in Figure 18 is directly applied to the code developed to implement the proposed enhanced MP scheme. The delayed time parameter $T$ is set to $2.8 \mathrm{~ns}$. 




FIGURE 11: Impulse response of the single band CPW antenna.

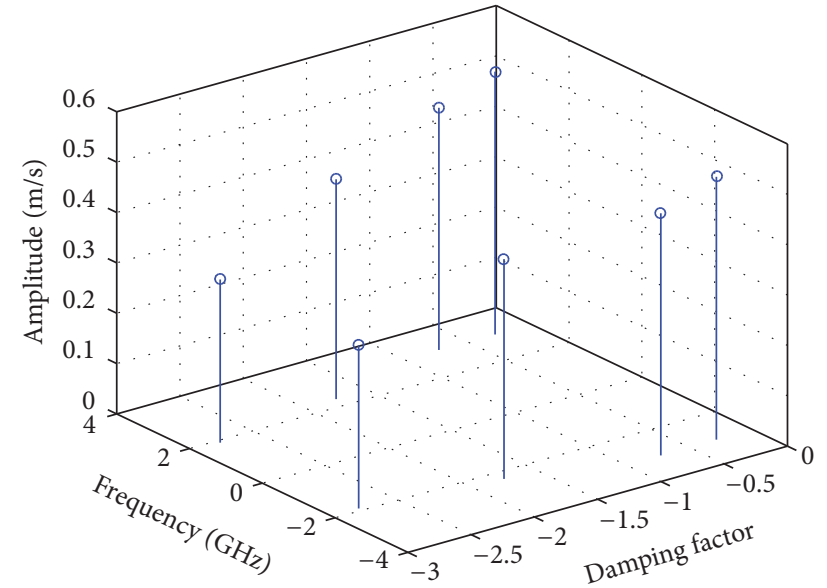

(a)



(b)

FIGURE 12: Using classical MP method: (a) pole-residue relationship and (b) frequency versus damping factor.

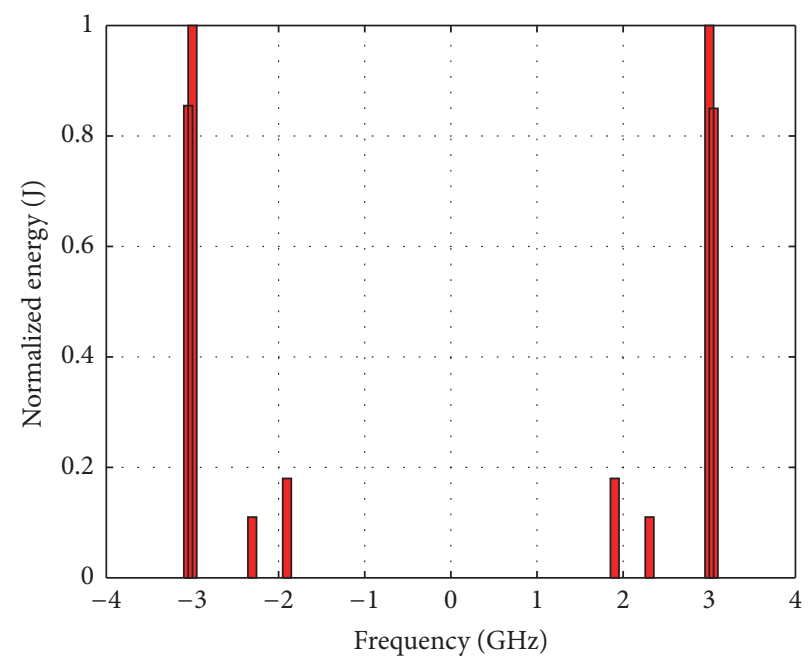

Figure 13: Pole normalized $E$ levels with frequency. 


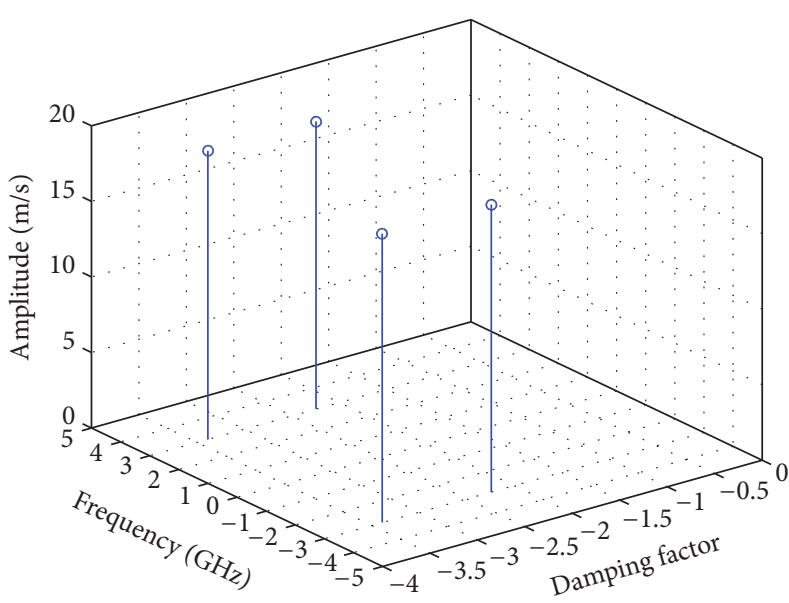

(a)

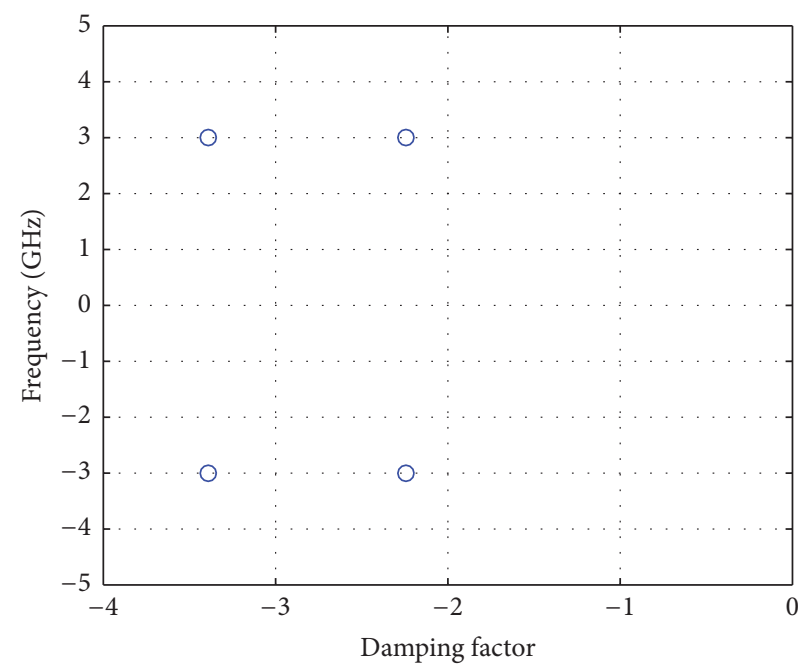

(b)

FIGURE 14: Using proposed enhanced MP method: (a) pole-residue relationship and (b) frequency versus damping factor.

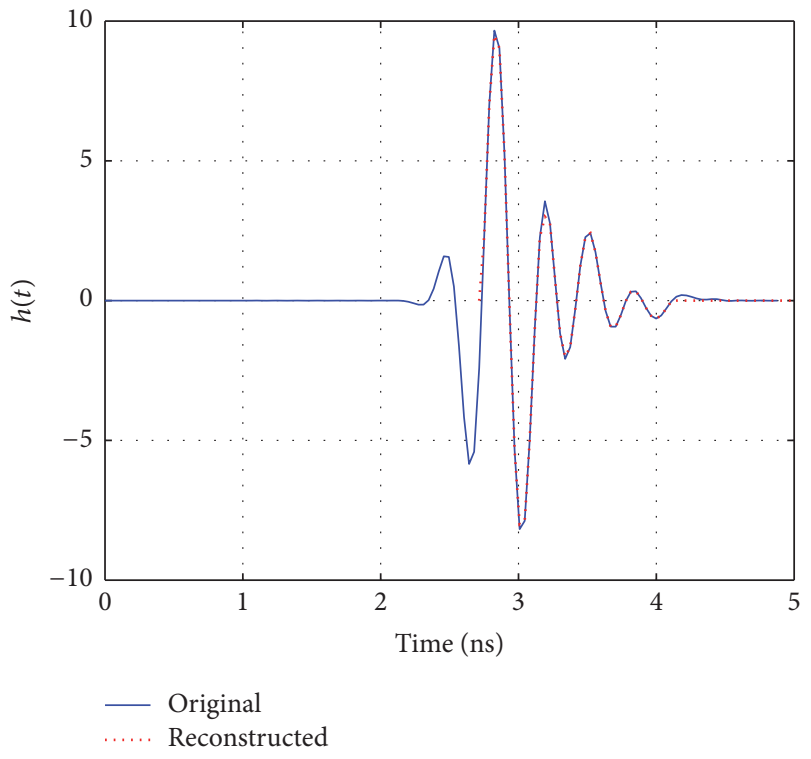

FIGURE 15: Comparison of the original impulse response and the reconstructed response of the CPW antenna.

The pole-residue relationship with frequency as a function of the damping factor is plotted in Figures 21(a) and 21(b), respectively.

The results in Figure 21 show that all of the low $E$ nonphysical poles in the antenna response are eliminated. There is one pair of poles located at about $2.3 \mathrm{GHz}$. The extracted poles are well within the resonance band of the narrow band antenna. To validate the extracted poles, the impulse response of the antenna is reconstructed using SEM (1). Comparison of the original impulse response and the reconstructed response is shown in Figure 22 where the reconstructed response resembles the original impulse response.



FIGURE 16: Geometry of the microstrip antenna.

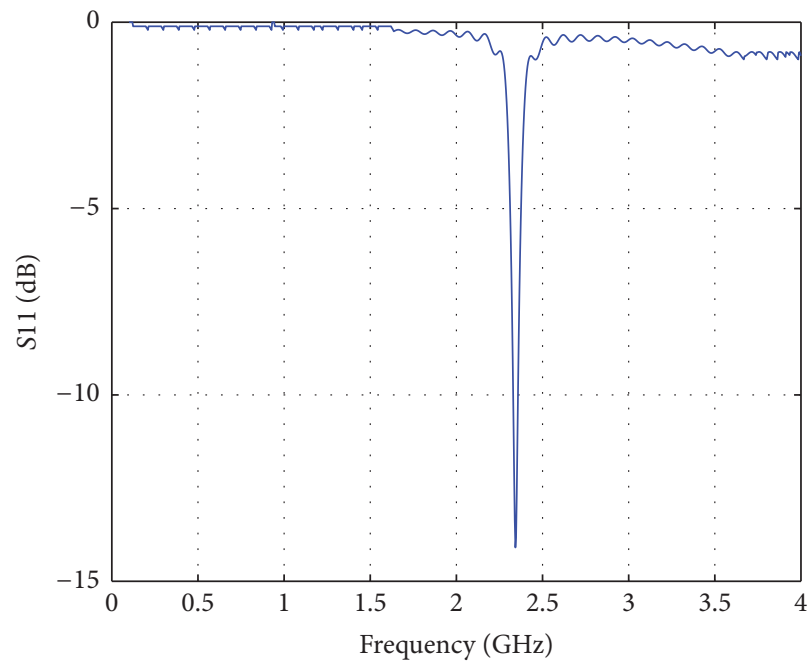

FIGURE 17: $S_{11}$ of the single band microstrip antenna. 


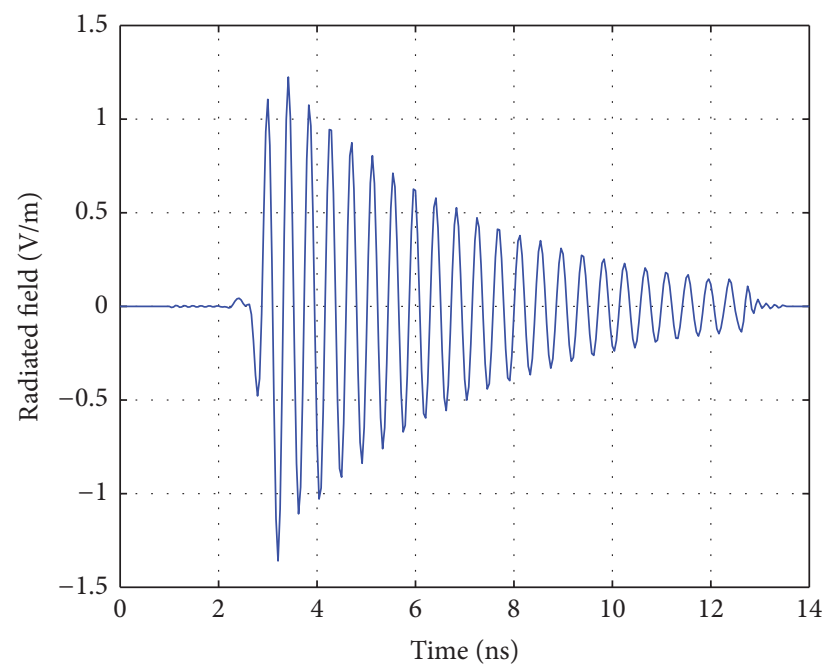

FIGURE 18: Impulse response of the single band microstrip antenna.

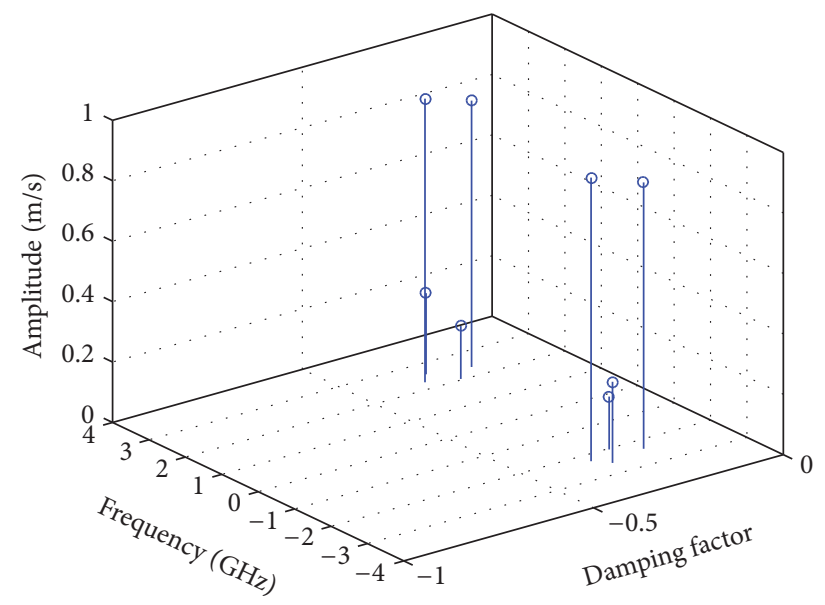

(a)



(b)

FIGURE 19: Using classical MP method: (a) pole-residue relationship and (b) frequency versus damping factor of microstrip antenna.

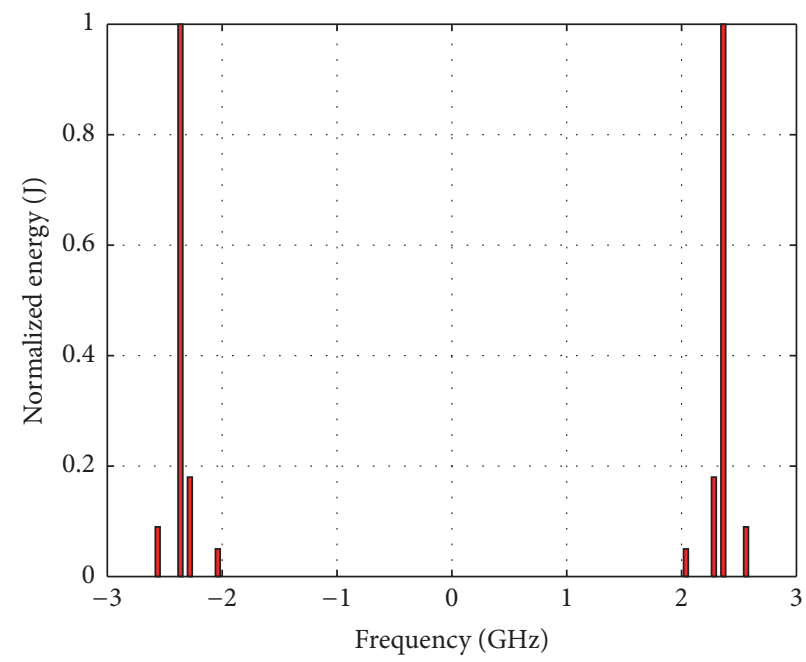

Figure 20: Pole normalized $E$ levels with frequency. 


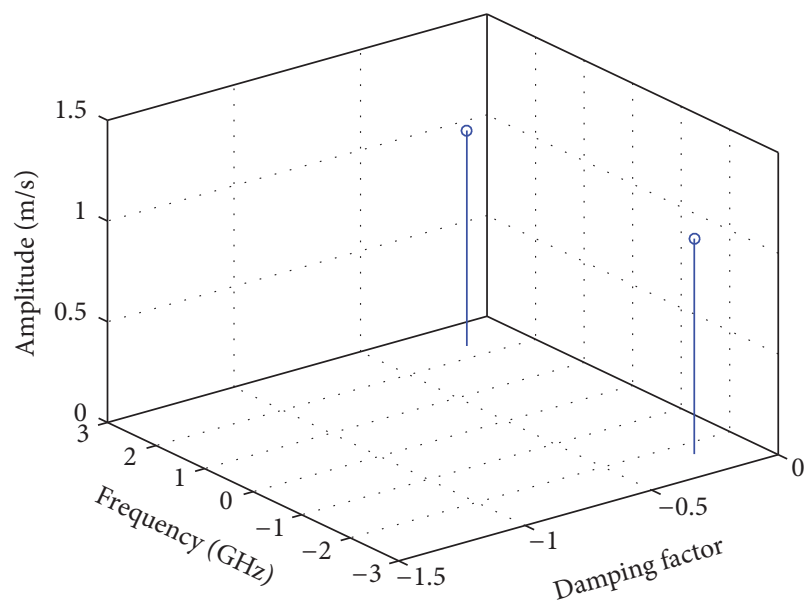

(a)

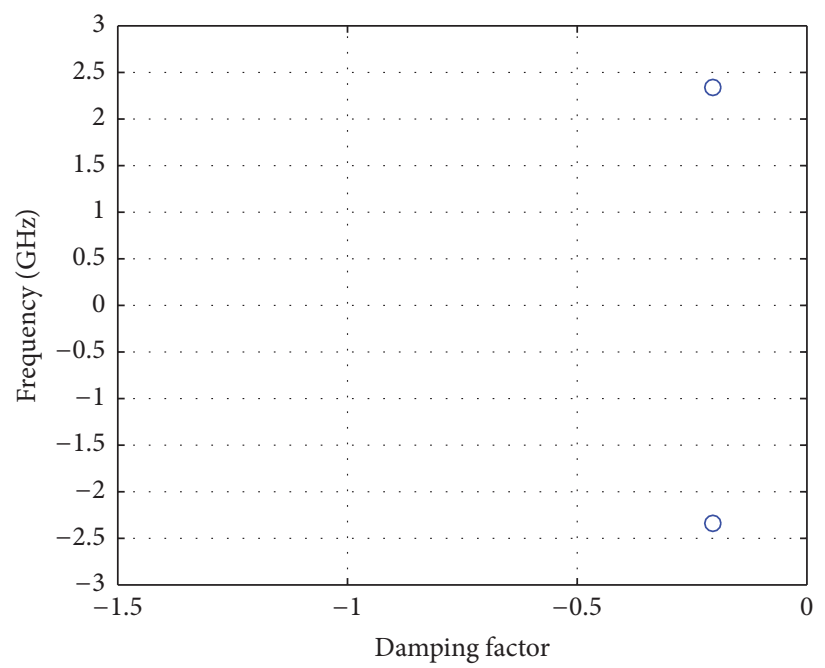

(b)

FIGURE 21: Using proposed enhanced MP method: (a) pole-residue relationship and (b) frequency versus damping factor.

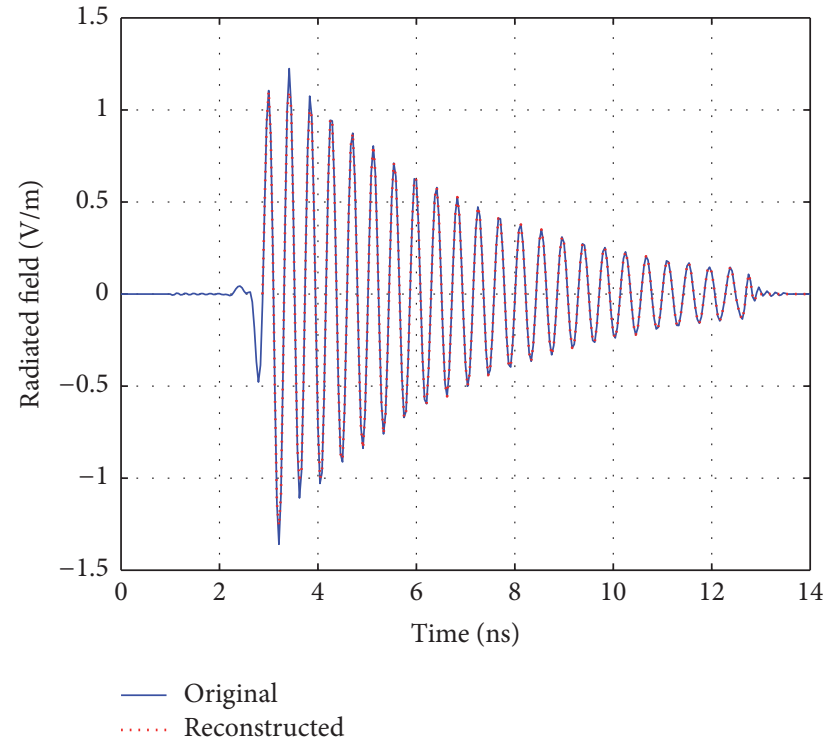

FIGURE 22: Comparison of original impulse response and the reconstructed response of the microstrip antenna.

\section{Measurement Results}

To support the analysis, the microstrip antenna, investigated in Section 4.3 and shown in Figure 16, is fabricated on a substrate with dielectric constant of 4.3 and height of $0.78 \mathrm{~mm}$. The photograph of the fabricated antenna is shown in Figure 23.

Comparison of the measured and the simulated $S_{11}$ for this antenna is shown in Figure 24.

The measured impulse response of the fabricated antenna is obtained using the Geozondas time domain antenna measurement systems setup [15]. A Shielded Loaded BowTie Antenna with balun AU-UWB is used as a transmitting



FIGURE 23: Photograph of single band microstrip antenna.

antenna while the fabricated microstrip antenna is the antenna under test [15]. The distance between antennas is about $2.8 \mathrm{~m}$. The Pulse Generator Mainframe GZ1106DL2 has been used to generate a Gaussian pulse with a width of about $0.4 \mathrm{~ns}$. The measured impulse response of the microstrip antenna shown in Figure 26 is directly applied to the developed enhanced MP method. The pole-residue relationship and the frequency as a function of the damping factor are plotted in Figures 25(a) and 25(b), respectively. Poles with sufficiently low energy as compared to 


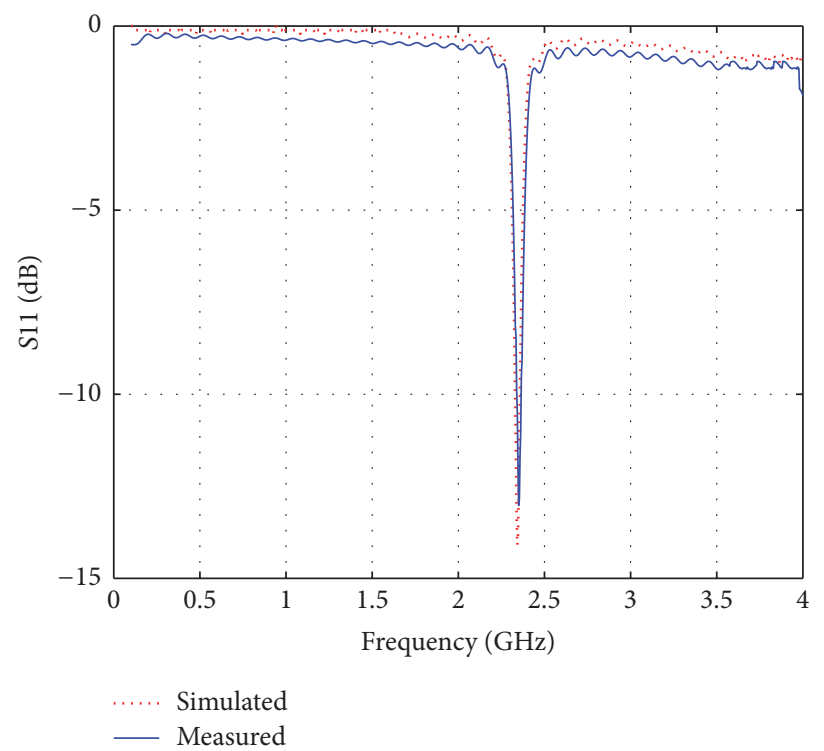

FIgURE 24: Comparison of measured and simulated $S_{11}$.

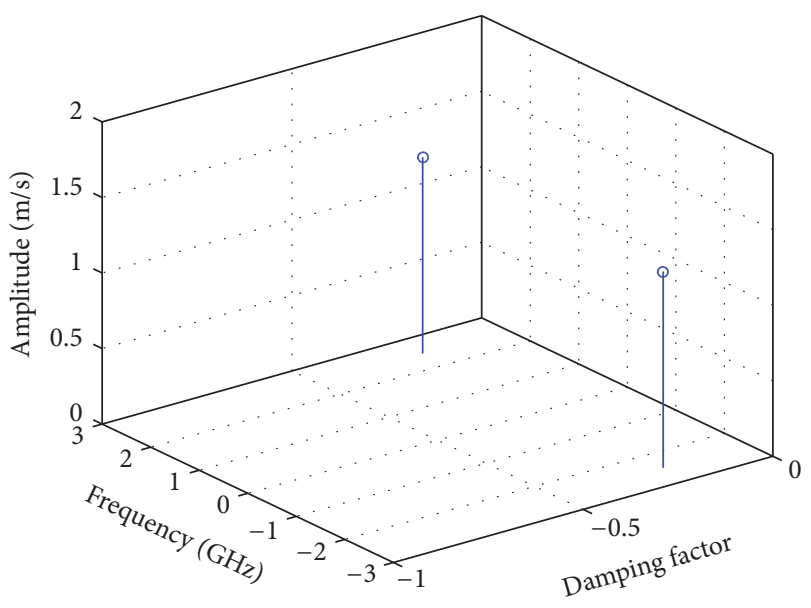

(a)

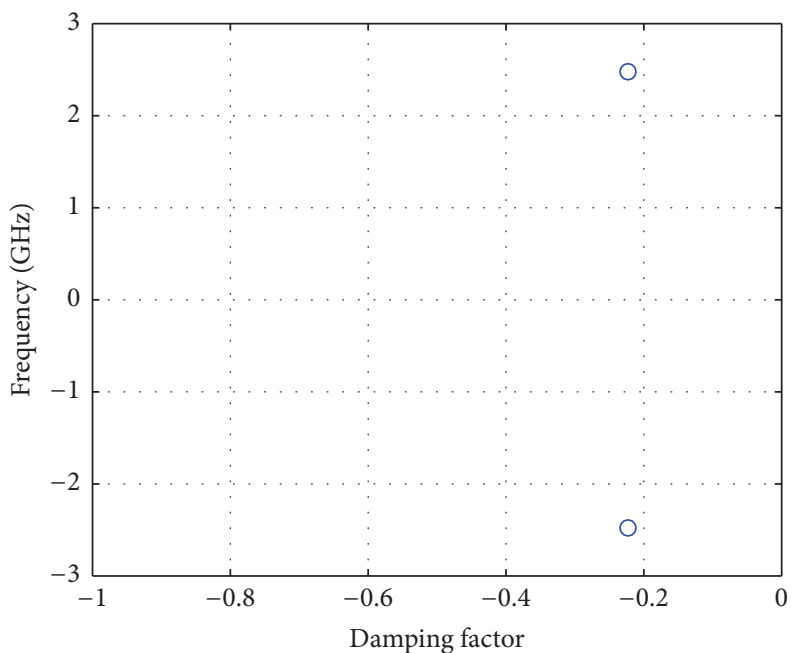

(b)

FIGURE 25: Using proposed enhanced MP method: (a) pole-residue relationship and (b) frequency versus damping factor.

the resonant poles are appeared as nonphysical poles. The results in Figure 25 show that there is one pair of poles located at about $2.3 \mathrm{GHz}$. The extracted poles are well within the resonance band of the narrow band antenna. To validate the extracted poles, the impulse response of the antenna is reconstructed. Comparison of the original measured impulse response and the reconstructed response is shown in Figure 26 where the reconstructed response matched very well with the original measured impulse response.

\section{Conclusions}

This paper presented time domain and frequency domain system modeling of three narrow band antennas using the singularity expansion method (SEM). To acquire the accurate physical complex poles and the corresponding residues of the narrow band antenna model, an enhanced Matrix Pencil (MP) method has been applied to the impulse response of the antennas. An additional time parameter $T$ is used to acquire the late time response of the antenna to extract accurate physical poles of narrow band antennas. The poles extracted by the enhanced MP method are located precisely within the single operating band of each antenna and the reconstructed far field impulse responses superbly match the original impulse responses of the antennas. A narrow band microstrip antenna has been fabricated and measured to demonstrate the developed concept. The far field impulse response of the fabricated antenna is measured, system-modeled using the proposed method, and then reconstructed correctly using the SEM based model. 


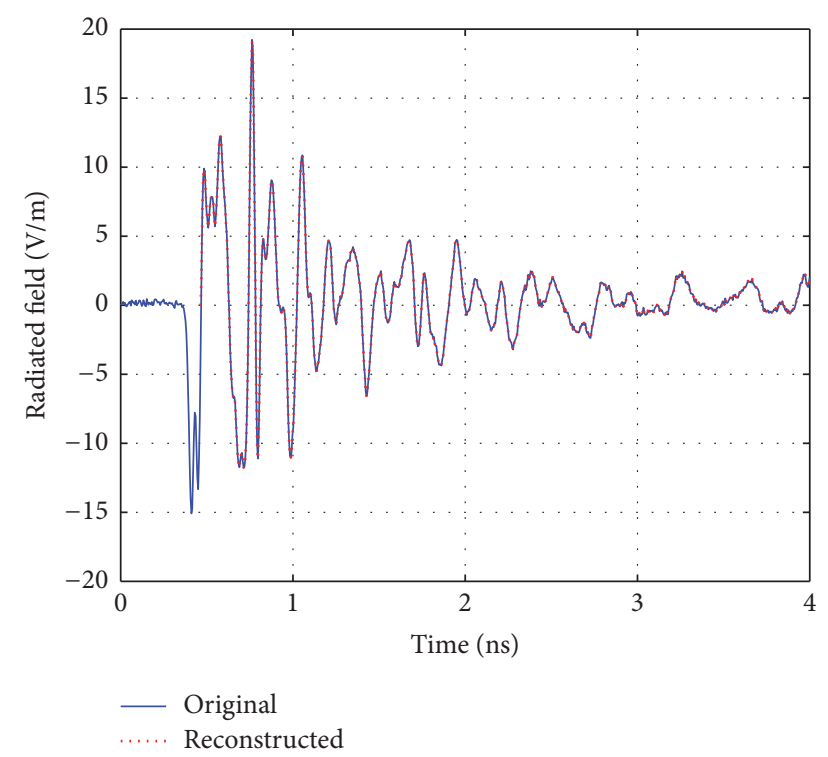

FIGURE 26: Comparison of the original measured impulse response and the reconstructed response of the microstrip antenna.

\section{Conflicts of Interest}

The authors declare that there are no conflicts of interest regarding the publication of this paper.

\section{Acknowledgments}

The authors would like to thank the Deanship of the Scientific Research and the Research Center at the College of Engineering, King Saud University, Riyadh, Saudi Arabia.

\section{References}

[1] C. E. Baum, "The singularity expansion method: background and developments," Electromagnetics, vol. 1, no. 4, pp. 351-360, 1981.

[2] C. E. Baum, E. J. Rothwell, K.-M. Chen, and D. P. Nyquist, "The singularity expansion method and its application to target identification," Proceedings of the IEEE, vol. 79, no. 10, pp. 14811492, 1991.

[3] C. E. Baum, "Discrimination of buried targets via the singularity expansion," Inverse Problems, vol. 13, no. 3, pp. 557-570, 1997.

[4] F. Sarrazin, P. Pouliguen, A. Sharaiha, J. Chauveau, and P. Potier, "Antenna physical poles extracted from measured backscattered fields," IEEE Transactions on Antennas and Propagation, vol. 63, no. 9, pp. 3963-3972, 2015.

[5] C. E. Baum, "On the singularity expansion method for the solution of electromagnetic interaction problems," DTIC Document, 1971.

[6] M. Manteghi, D. B. Cooper, and P. P. Vlachos, "Application of singularity expansion method for monitoring the deployment of arterial stents," Microwave and Optical Technology Letters, vol. 54, no. 10, pp. 2241-2246, 2012.

[7] F. Sarrazin, J. Chauveau, P. Pouliguen, P. Potier, and A. Sharaiha, "Accuracy of singularity expansion method in time and frequency domains to characterize antennas in presence of noise,"
IEEE Transactions on Antennas and Propagation, vol. 62, no. 3, pp. 1261-1269, 2014.

[8] S. Licul and W. A. Davis, "Unified frequency and time-domain antenna modeling and characterization," IEEE Transactions on Antennas and Propagation, vol. 53, no. 9, pp. 2882-2888, 2005.

[9] A. Roussafi, N. Fortino, and J.-Y. Dauvignac, "UWB antenna 3D characterization using matrix pencil method," in Proceedings of the IEEE Conference on Antenna Measurements and Applications (CAMA '14), pp. 1-4, November 2014.

[10] T. K. Sarkar and O. Pereira, "Using the matrix pencil method to estimate the parameters of a sum of complex exponentials," IEEE Antennas and Propagation Magazine, vol. 37, no. 1, pp. 4855, 1995.

[11] R. Rezaiesarlak and M. Manteghi, Chipless RFID: Design Procedure and Detection Techniques, Springer, 2014.

[12] H.-S. Lui and N. V. Z. Shuley, "Evolutions of partial and global resonances in transient electromagnetic scattering," IEEE Antennas and Wireless Propagation Letters, vol. 7, pp. 436-439, 2008.

[13] E. J. Rothwell, K. M. Chen, and D. P. Nyquist, "An adaptivewindow-width short-time Fourier transform for visualization of radar target substructure resonances," IEEE Transactions on Antennas and Propagation, vol. 46, no. 9, pp. 1393-1395, 1998.

[14] Computer Simulation Technology, CST Microwave Studio, Framingham, Mass, USA, 2015.

[15] GEOZONDAS, "Time domain antena measurement system," http://www.geozondas.com/far_field.php. 


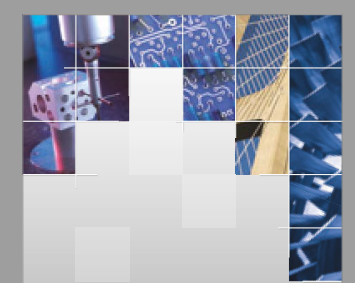

\section{Enfincering}
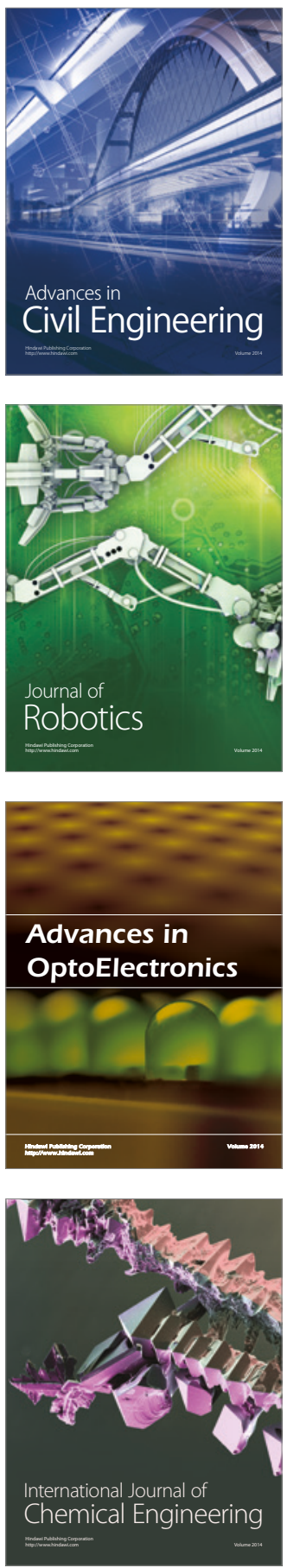

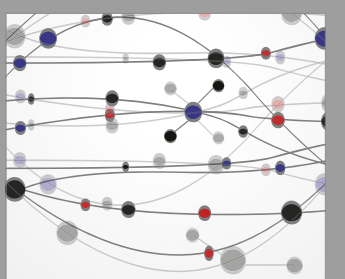

The Scientific World Journal

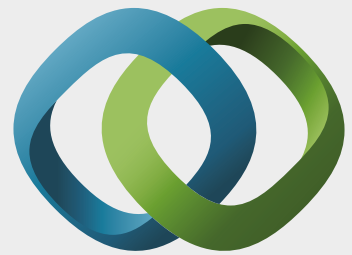

\section{Hindawi}

Submit your manuscripts at

https://www.hindawi.com
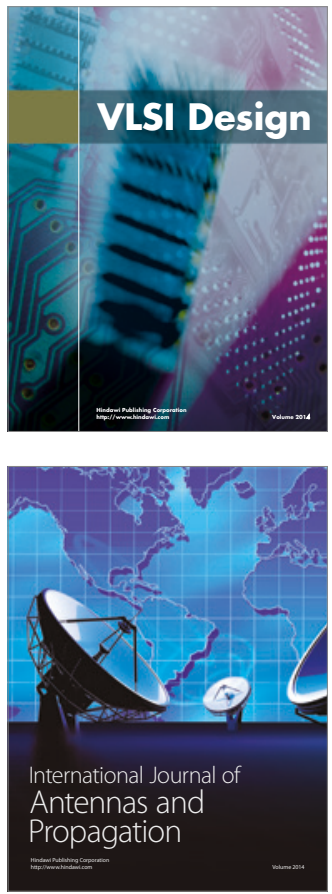

\section{Rotating}

Machinery
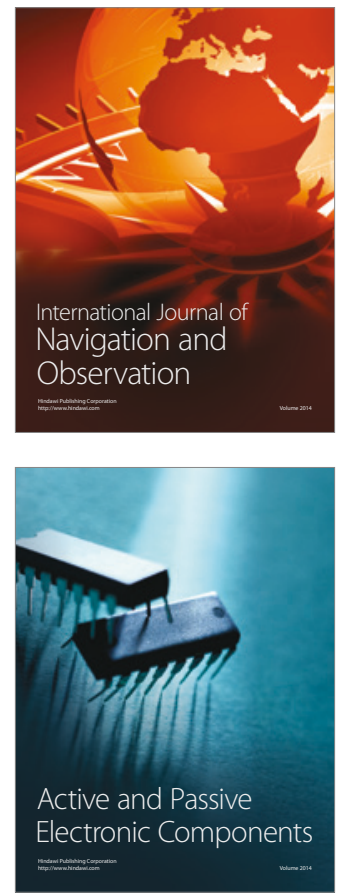
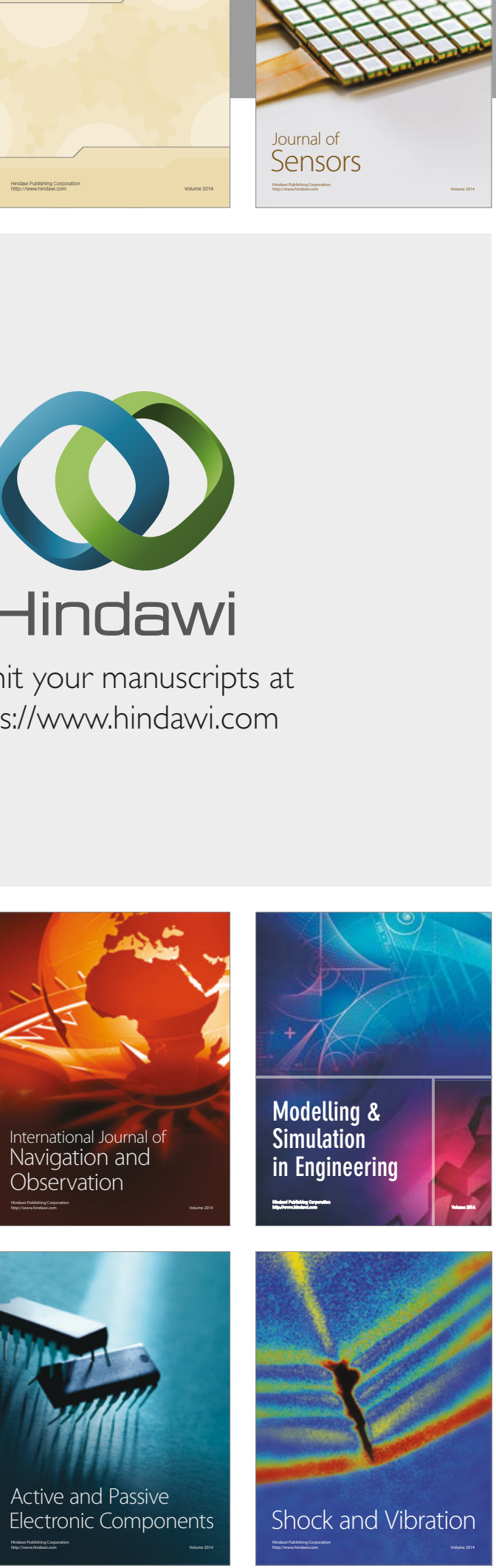
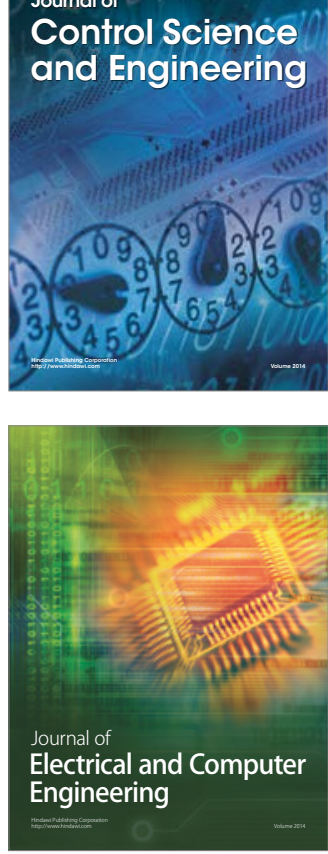

Distributed

Journal of

Control Science

and Engineering
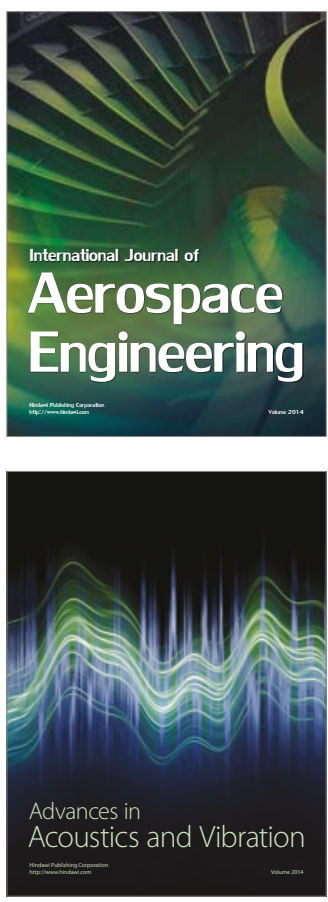

Sensor Networks 